\title{
Facing Novel Challenges in Neurodegenerative Diseases Drug Discovery: From Small Molecules to Targeted Therapies ${ }^{\dagger}$
}

\author{
Maria João Matos $1, *$, Dolores Viña ${ }^{2}$, Lourdes Santana ${ }^{1}$ and Eugenio Uriarte ${ }^{1,3}$ \\ 1 Departamento de Química Orgánica, Facultad de Farmacia, Universidade de Santiago de Compostela, \\ 15782 Santiago de Compostela, Spain \\ 2 Centro de Investigación en Medicina Molecular y Enfermedades Crónicas (CIMUS), Universidade de \\ Santiago de Compostela, 15782 Santiago de Compostela, Spain \\ 3 Instituto de Ciencias Químicas Aplicadas, Universidad Autónoma de Chile, 7500912 Santiago, Chile \\ * Correspondence: mariacmatos@gmail.com or mariajoao.correiapinto@usc.es \\ + Presented at the 2nd Molecules Medicinal Chemistry Symposium (MMCS): Facing Novel Challenges in \\ Drug Discovery, Barcelona, Spain, 15-17 May 2019.
}

Published: 7 August 2019

Keywords: Small molecules; nanoparticles; protein-conjugates; targeted therapies; Parkinson's disease

Neurodegenerative diseases are becoming increasingly prevalent with the aging of the general population. The twentieth century witnessed a significant demographic change in the human population of the industrialized world that is currently followed by a similar shift of life expectancy to upper age ranges in developing countries. The effectiveness of a drug depends on accumulation at the site of action at therapeutic levels. However, challenges such as rapid renal clearance, degradation or non-specific accumulation require drug delivery enabling technologies. Targeted drug delivery is a very promising concept, which still needs improvement for better clinical outcomes. Understanding some of the molecular changes associated to these ubiquitous and widespread diseases has stimulated efforts to develop drugs that specially target key proteins. Protein behavior is scrupulously regulated by a plethora of post-translational modifications (PTMs). It is therefore desirable to develop methods to design rational PTMs to modulate specific protein functions [1-3].

We report different approaches and illustrate their successful implementation in the search for treatment for Parkinson's disease. Computer-assisted design of potent small molecules, together with the development of new carriers, allows a wide range of possibilities for targeted therapies. Knowledge on biochemical processes brings the opportunity to provide treatments that are potentially less toxic and more effective than traditional therapeutic approaches.

Acknowledgements: This work was supported by the University of Santiago de Compostela (2018-PU067). Authors would like to thank Xunta da Galicia Plan of Research, Innovation and Growth 2011-2015 (Plan I2C, ED481B 2014/086-0 and ED481B 2018/007) for the financial support.

\section{References}

1. Matos, M.J.; Oliveira, B.L.; Martínez-Sáez, N.; Guerreiro, A.; Cal, P.M.S.D.; Bertoldo, J.; Maneiro, M.; Perkins, E.; Howard, J.; Deery, M.J.; et al. Chemo- and Regioselective Lysine Modification on Native Proteins. J. Am. Chem. Soc. 2018, 140, 4004-4017.

2. Matos, M.J. Learning from Nature: the Role of Albumin in Drug Delivery. Fut. Med. Chem. 2018, 10, 983985. 
3. Matos, M.J.; Labão-Almeida, C.; Sayers, C.; Dada, O.; Tacke, M.; Bernardes, D.G.J.L. Synthesis and Biological Evaluation of Homogeneous Thiol-Linked $\mathrm{NHC}^{*}$-Au-Albumin and -Trastuzumab Bioconjugates. Chem. Eur. J. 2018, 24, 12250-12253.

(c) (1)

(C) 2019 by the authors. Licensee MDPI, Basel, Switzerland. This article is an open access article distributed under the terms and conditions of the Creative Commons Attribution (CC BY) license (http://creativecommons.org/licenses/by/4.0/). 